CARNETS DE Carnets de géographes

GÉOGRAPHES.

$8 \mid 2015$

Géographie(s) de la lenteur

\title{
La production capitaliste des logements à Bruxelles
}

Promotion immobilière et division sociale de l'espace

\section{Alice Romainville}

\section{(2) OpenEdition}

Journals

Édition électronique

URL : http://journals.openedition.org/cdg/338

DOI : $10.4000 /$ cdg.338

ISSN : 2107-7266

Éditeur

UMR 245 - CESSMA

\section{Référence électronique}

Alice Romainville, "La production capitaliste des logements à Bruxelles », Carnets de géographes [En

ligne], 8 | 2015, mis en ligne le 01 septembre 2015, consulté le 23 septembre 2020. URL : http://

journals.openedition.org/cdg/338; DOI : https://doi.org/10.4000/cdg.338

Ce document a été généré automatiquement le 23 septembre 2020.

\section{(c)}

La revue Carnets de géographes est mise à disposition selon les termes de la Licence Creative

Commons Attribution - Pas d'Utilisation Commerciale - Pas de Modification 4.0 International. 


\section{La production capitaliste des logements à Bruxelles}

Promotion immobilière et division sociale de l'espace

\section{Alice Romainville}

1 Dans le système de production de l'habitat qui existe à Bruxelles, en ce début de $21^{\mathrm{e}}$ siècle, la production de logements est majoritairement laissée à des sociétés privées. Sur le marché du logement se rencontrent une demande et une offre, des «consommateurs» et des producteurs de logements, dont les intérêts sont diamétralement opposés.

2 Cette étude aborde la problématique de la production de logements dans la Région de Bruxelles-Capitale, la partie centrale de l'agglomération fonctionnelle de Bruxelles. Sur ce territoire de $161 \mathrm{~km}^{2}$ vit un dixième de la population belge. On y observe, au cours de la première décennie 2000, un niveau élevé de production de logements en même temps qu'une pénurie de logements abordables aux conséquences sociales dévastatrices. Les statistiques basées sur les ventes de logements indiquent un doublement des prix entre 2000 et 2010, alors que le revenu moyen des ménages n'évolue pour ainsi dire pas (voir figure 1). 
Figure 1. Évolution des prix de vente et des revenus

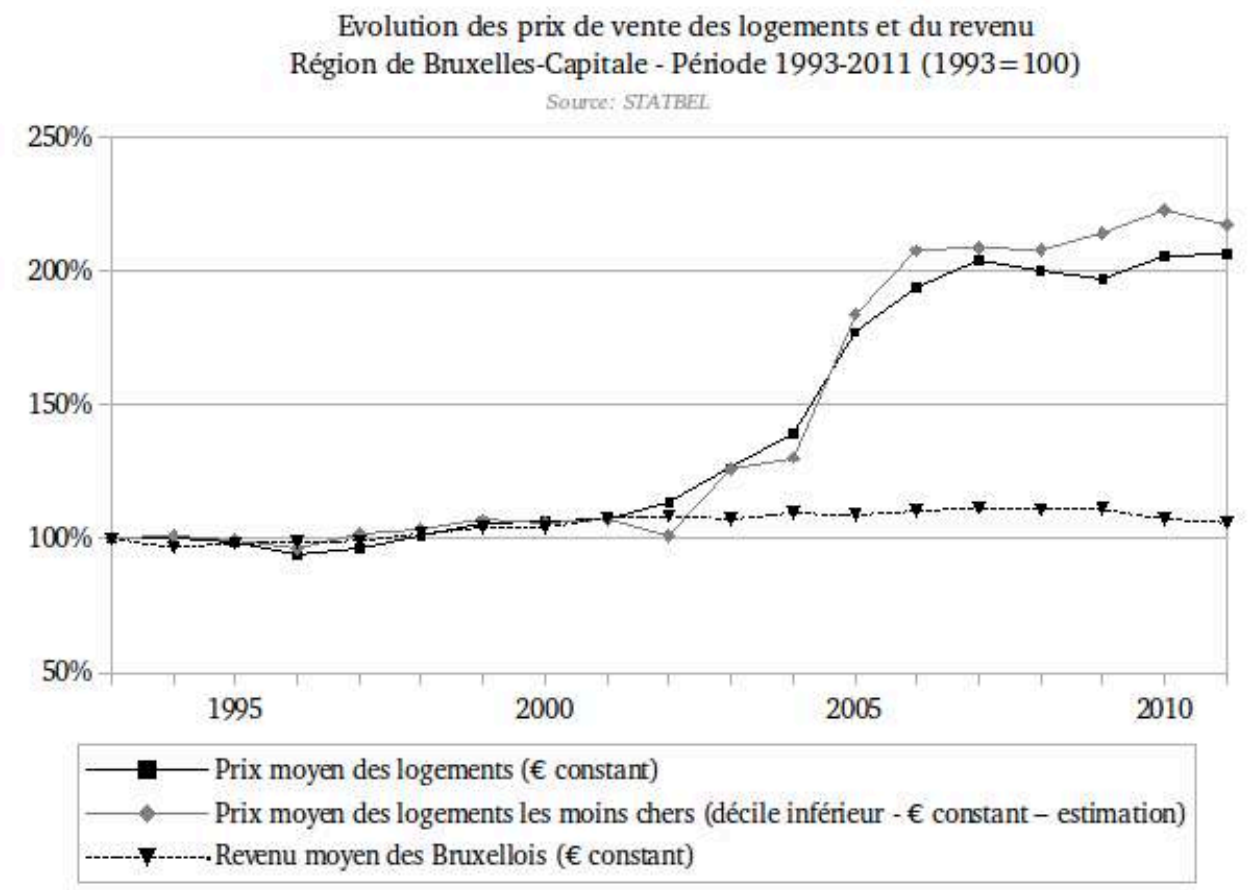

Source : STATBEL (DG Statistique)

3 Cette étude lève le voile sur les mécanismes à l'œuvre du côté des sociétés qui sont les agents principaux de la production capitaliste de logements. Ces sociétés sont celles qui assurent, dans le domaine résidentiel, la fonction de promoteur immobilier (une fonction assurée par des sociétés de toutes sortes, qui n'ont pas toutes officiellement la promotion immobilière comme activité principale).

4 Au départ de la recherche, il y a d'abord l'objectif de décrire les sociétés privées qui produisent des logements à Bruxelles et de comprendre leur mode de fonctionnement commun.

5 La deuxième question à laquelle l'analyse a tenté de répondre est celle de la façon dont ces sociétés utilisent, sont contraints par, et éventuellement transforment, la division sociale de l'espace: qu'est-ce qui détermine les choix des sociétés, en termes de quartiers et de logements produits ? Quels facteurs vont pousser les sociétés à investir dans des quartiers chers, ou au contraire dans des quartiers bon marché ? À produire des logements dans la même gamme de prix que les autres logements du quartier, ou au contraire à cibler une clientèle différente de la population en place?

6 Les théories et concepts utilisés dans cette étude (concernant notamment le système de la promotion immobilière, la fixation des prix des terrains, la gentrification) sont issus de textes qui peuvent, pour l'essentiel, être rangés au rayon des théories urbaines critiques.

7 L'essentiel du matériel empirique de recherche, lui, est constitué de bases de données qualitatives et quantitatives. Des données relatives aux permis d'urbanisme (le " permis de construire » bruxellois) octroyés à des sociétés ont été complétées par des données concernant ces sociétés, afin de constituer une base de données mettant en relation les projets de logements et les sociétés ayant investi dans ces projets (promoteurs et investisseurs). Ces sociétés sont notamment caractérisées selon l'origine des capitaux 
qu'elles mobilisent, grâce à l'identification des participations financières qui les lient à d'autres sociétés (actionnaires, filiales). Les informations collectées au sujet des sociétés productrices de logements permettent d'étudier les liens entre ce secteur et le reste du système économique, son degré de financiarisation, son degré d'ouverture aux capitaux étrangers.

8 Par ailleurs, 13 entretiens ont été menés auprès de professionnels du secteur. Ces entretiens ont permis d'éclairer les pratiques des promoteurs (pourquoi tel projet à tel endroit? Pourquoi tel partenaire financier plutôt que tel autre ?) et d'illustrer le texte avec des exemples concrets.

9 La partie théorique de l'étude expose la façon dont les promoteurs immobiliers peuvent maximiser leur taux de profit à travers la localisation de leurs opérations de logements. Elle fait le lien entre les pratiques observées des promoteurs, la théorie de la rente foncière, et la question de la reproduction / transformation de la division sociale de l'espace. L'analyse détaillée des calculs économiques opérés par les promoteurs immobiliers montre que le système de la promotion immobilière est fortement déterminé par des éléments qui lui sont extérieurs : la conjoncture et les politiques économiques, les politiques publiques en matière d'habitat et d'aménagement du territoire, mais aussi la division sociale de l'espace urbain. Celle-ci s'impose aux promoteurs et les contraint, par l'intermédiaire du prix de vente maximum des logements et du tribut prélevé par les vendeurs de terrains. Cette analyse permet aussi de constater que le fait que la production capitaliste de logements ne réponde pas aux besoins en logements bon marché résulte, en réalité, d'une impossibilité économique.

La seconde partie de l'étude, plus empirique, décrit les promoteurs actifs en Région bruxelloise, par l'intermédiaire d'une typologie et de portraits d'entreprises.

Elle compare ensuite les stratégies spatiales de ces promoteurs, en analysant le nombre de logements créés par les différents types de promoteurs dans différents types de quartiers (voir figure 2). 
Figure 2. Typologie spatiale

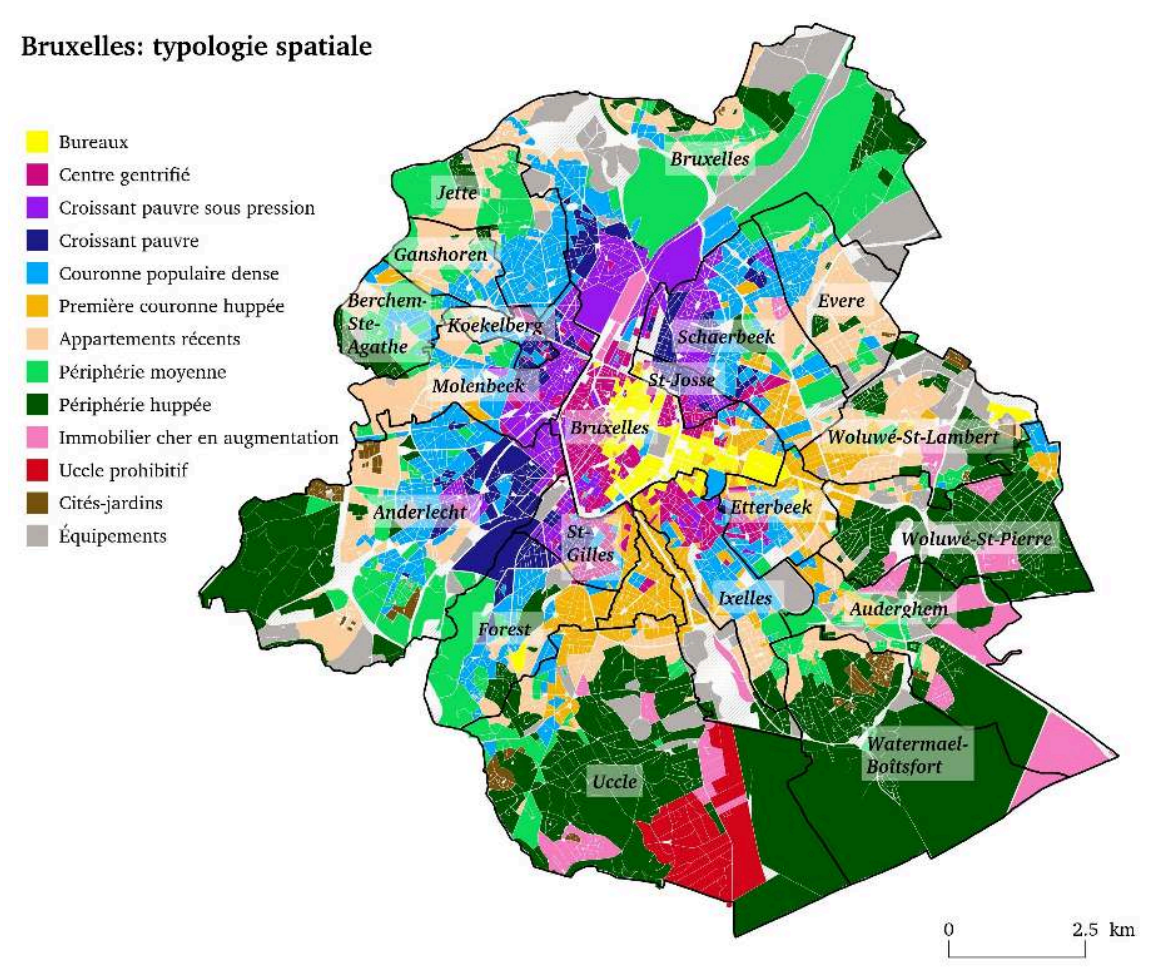

12 On y apprend que les choix de localisation sont fortement influencés par la taille des sociétés (voir figure 3) et par l'origine (géographique et sectorielle) des capitaux investis.

Figure 3. Géographie comparée des opérations selon la taille des promoteurs. Source : données relatives aux permis d'urbanisme (Bruxelles Développement Urbain) et exploitation complémentaire

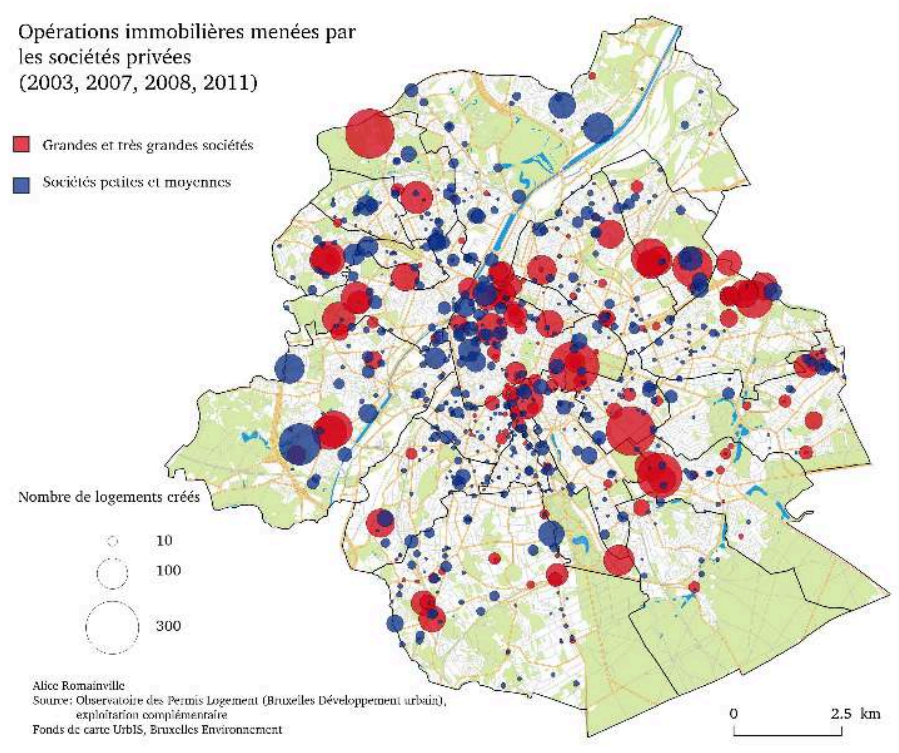


Ces choix de localisation correspondent à différentes stratégies de maximisation du taux de profit. Par exemple, les "promoteurs-entrepreneurs" réalisent, pour faire tourner au maximum la capacité technique de leur pôle construction, de grandes opérations pour lesquelles ils se «contentent» de prix moyens. Les promoteurs issus du secteur financier, eux, réalisent de grandes opérations, mais surtout dans les quartiers chers (zones de bureaux, périphérie huppée). Les quartiers densément peuplés, y compris les quartiers en gentrification, constituent plutôt le domaine des petits promoteurs locaux.

\section{Thèse disponible en ligne}

http://hdl.handle.net/2013/ULB-DIPOT:oai:dipot.ulb.ac.be:2013/209049

\section{Discipline \\ Géographie}

\section{Université}

Université Libre de Bruxelles

\section{Directeur}

Jean-Michel Decroly

Membres du jury de thèse, soutenue en juin 2015

Mathieu Van Criekingen, géographe, professeur, Université Libre de Bruxelles, président du jury

Christian Kesteloot, géographe, professeur, Université Libre de Bruxelles et Katholieke Universiteit Leuven, secrétaire du jury

Christian Vandermotten, docteur en sciences géographiques et licencié en urbanisme, professeur, Université Libre de Bruxelles

Loïc Bonneval, sociologue, maitre de conférences, Université de Lyon II

Daniel Tranchida, docteur en géographie, Université Paul Valéry

\section{Courriel}

alice.romainville [at] ulb.ac.be

INDEX

Thèmes : Carnets de soutenances 